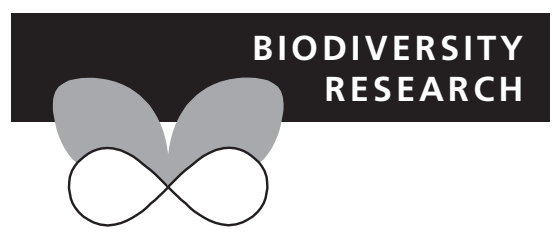

\title{
Modelling species distributional shifts across broad spatial extents by linking dynamic occupancy models with public-based surveys
}

\author{
Truly Santika ${ }^{1,2 \star}$, Clive A. McAlpine ${ }^{1,2}$, Daniel Lunney ${ }^{3,4}$,
} Kerrie A. Wilson ${ }^{2,5}$ and Jonathan R. Rhodes ${ }^{1,2}$

${ }^{1}$ School of Geography, Planning and Environmental Management, The University of Queensland, Brisbane, QLD 4072, Australia, ${ }^{2}$ NERP Environmental Decisions $H u b$, The University of Queensland, Brisbane, QLD 4072, Australia, ${ }^{3}$ Office of Environment and Heritage NSW, PO Box 1967, Hurstville, NSW 2220, Australia, ${ }^{4}$ School of Veterinary and Life Sciences, Murdoch University, Perth, WA 6150, Australia, ${ }^{5}$ School of Biological Sciences, The University of Queensland, Brisbane, QLD 4072, Australia

${ }^{\star}$ Correspondence: Truly Santika, School of Geography, Planning and Environmental Management, The University of Queensland, Brisbane, QLD 4072, Australia.

E-mail: trulysantika@gmail.com

\begin{abstract}
Aim To understand climate and landscape drivers of species distributional shifts across broad spatial extents by integrating dynamic occupancy models with distribution data collected from the public.
\end{abstract}

Location New South Wales (NSW), Australia.

Methods We used data on koala (Phascolarctos cinereus) presence and absence collected across the state of NSW from public surveys between 1987 and 2011. A dynamic occupancy model was built to quantify the role of climate and land use change on koala extinction risk and occupancy. We contrasted the model results against the more usual static occupancy model approach. We then developed scenarios of future climate, land clearing and urbanization and predicted the distribution of the koalas over the next 20 years based on the dynamic occupancy model.

Results The static model indicates koala occupancy in 1987 and in 2011 depended most strongly on annual rainfall and distance to water features. Housing density and its interaction with Eucalyptus forest cover only minimally affected koala occupancy. However, for the dynamic occupancy model, extinction risk (the metric of dominant concern for species conservation) depended most strongly on Eucalyptus forest cover and its interaction with housing density, while annual rainfall only minimally affected extinction risk. We predicted extinction risk to be higher in western NSW than in the east and that extinction risk may increase under future scenarios of climate and land use change.

Main conclusions This study underlines the importance of incorporating extinction dynamics when modelling species distributional shifts under climate and land use change and we provide an approach for doing so using publicbased surveys. As conservation objectives usually aim to maximize persistence, this is likely to lead to more reliable identification of conservation priorities than using static species distribution models. Combining public-based surveys and dynamic occupancy models provides a powerful approach for achieving this across broad spatial extents, thus providing an alternative approach when field-based data collection is impractical.

\section{Keywords}

Climate change, dynamic occupancy models, dynamic threats, extinction risk, koala, land use change, multiple threats, public-based surveys, species distributional shift. 


\section{INTRODUCTION}

Threats to biodiversity posed by global change and multiple threats have made it imperative that the extinction risk of species be assessed to guide the conservation of biodiversity (Bellard et al., 2012; Mantyka-Pringle et al., 2012). Assessments of extinction risk are important because they provide information about the conservation status of species under threat. Furthermore, if assessments are spatial, they can inform management decisions regarding which areas require immediate conservation actions due to high levels of threat (Wilson et al., 2005). Despite its importance, species' extinction risk is often difficult to assess, especially spatially, as it requires comprehensive and long-term monitoring data across species' distributional ranges. For many species, such data are rarely available (Manley et al., 2004; Field et al., 2007).

To overcome the lack of long-term monitoring data over broad extents, conservation decisions often have to be informed by species' distribution models (Elith \& Leathwick, 2009) to predict species' responses to climate change, landscape change and other threats. These models typically rely on the relationships between the present-day species' occurrences and long-term climate predictors, and other non-climatic predictors such as land use to predict potential distributions of species under future scenarios (Pearson \& Dawson, 2003). These static types of models have been subject to criticism for failing to account for important dynamic processes influencing species' distributional change, such as colonization, extinction and dispersal (Thuiller et al., 2008). Ignoring dynamic processes may result in erroneous projections of future species' distributions (Zurell et al., 2009). Although the importance of accounting for dynamic processes has long been recognized (Mackey \& Lindenmayer, 2001), studies that explicitly account for dynamic processes in these models have only begun recently (Keith et al., 2008; Anderson et al., 2009). These studies typically integrate species' distribution models (Elith \& Leathwick, 2009) with population models (Moilanen et al., 1998), known as coupled or hybrid models (Conlisk et al., 2013).

An alternative is dynamic occupancy models (MacKenzie et al., 2003; Royle \& Kéry, 2007) that can be used to infer the occurrence of species and how dynamic processes, such as colonization and extinction, affect changes in species' occurrence over time (Martin et al., 2009). Factors, such as habitat conditions, climatic variables and data on threats driving these dynamic processes, are also able to be incorporated into these models (MacKenzie et al., 2003). Furthermore, these models make it possible to reliably estimate the proportion of the study extent occupied by a species, explicitly accounting for observation errors (Royle \& Kéry, 2007). However, there has been a lack of application of these methods to modelling species' distributional change across broad geographical extents, partly due to the fact that suitable survey data are often not readily available.
One solution is to use sightings of species, as reported by the general public, as an alternative source of data to assess species' distributional change over large spatial extents (Lunney et al., 2009). Well-designed surveys of the public can provide a credible, cost-effective alternative to traditional field surveys for direct observations or signs of a species (Devictor et al., 2010), especially for species that have a unique appearance and easily recognized, and are rare or difficult to detect otherwise (Van der Hoeven et al., 2004). The use of such data can facilitate the more general application of dynamic occupancy models to inform conservation decision-making, but have rarely been applied to the development of dynamic occupancy models (Kéry et al., 2010; Pillay et al., 2011).

We address this limitation by applying a dynamic occupancy model to public-based survey data on koala (Phascolarctos cinereus) presence and absence collected across the state of New South Wales (NSW), Australia. We show that this approach can be used to successfully quantify spatiotemporal changes in species' distributions and to identify multiple-threatening processes driving this change. In addition to the dynamic model, we constructed a static occupancy model as a comparison. We show that the dynamic model identifies quite different predictors driving species' distributional change when compared to the conventional static distribution model approach. Our method presents a practical approach for developing dynamic occupancy models across broad spatial extents to assess the potential impacts of climate change and anthropogenically driven land use change on species' distributions.

\section{METHODS}

\section{Study species and area}

The koala is an arboreal folivorous marsupial primarily restricted to the Eucalyptus forests of eastern and southern Australia and is one of Australia's most recognized native animals. Our study area was the state of NSW, Australia, covering an area of around $809,444 \mathrm{~km}^{2}$ (Fig. 1). In NSW, the koala is mainly distributed in the central and east coast regions (Reed et al., 1990; Lunney et al., 2009). However, their populations have been suffering rapid contractions due multiple threats (Lunney et al., 2009, 2010). The koala is listed as vulnerable under the Threatened Species Conservation (TSC) Act 1995 in NSW and under the Environmental Protection and Biodiversity Conservation (EPBC) Act 1999 at the Federal level. Nonetheless, no studies have attempted to model dynamic change in the distribution of the koala using spatio-temporal data over broad spatial extents. Such analyses are critical if we are to understand the conservation requirements of the species across its distribution.

\section{Data}

To develop the dynamic occupancy model, we used koala data collected via public-based surveys between 1987 and 


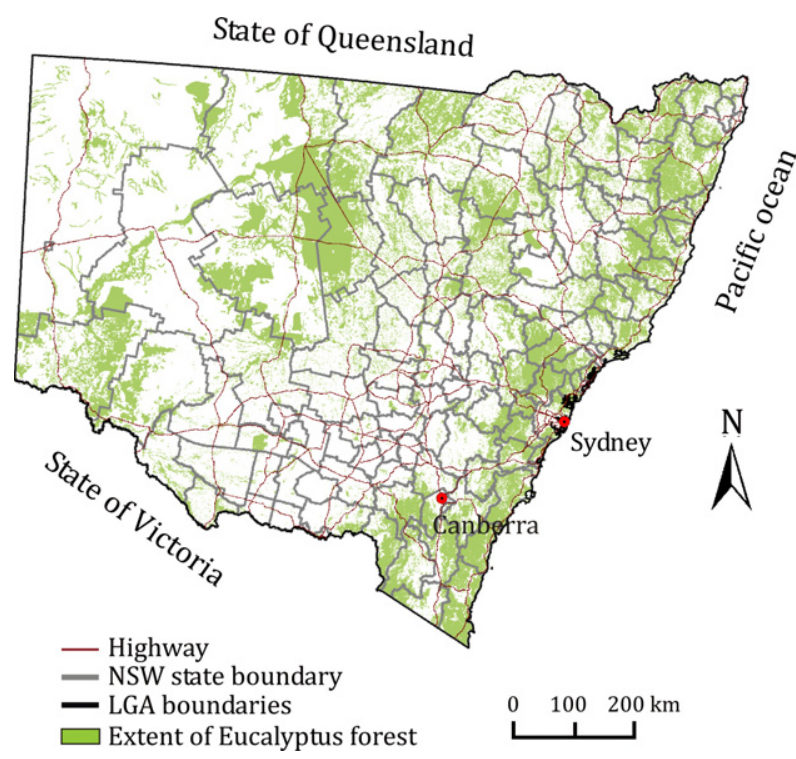

Figure 1 Study area in New South Wales (NSW), Australia, depicting major roads and highways, local government area (LGA) boundaries, and the extent of Eucalyptus forest.

2011 and seven environmental variables, including distance to water features, elevation, Eucalyptus forest cover, mean annual maximum summer temperature, mean annual rainfall, housing density and road density. Prior to analysis, we divided the state of NSW into grid cells of $10 \times 10 \mathrm{~km}^{2}$ in size and this was used as the resolution of analysis. These data are described below and in detail in Appendix S1 in the Supporting Information.

\section{Public-based survey data}

State-wide public-based surveys of the koala were undertaken in NSW as part of a national effort in 1987, 2006 and 20092011 (Reed et al., 1990; Lunney et al., 2009, in press). For the 1987 survey, respondents were asked whether they had seen koalas in their area, and where and when they saw the koalas. Thus, the 1987 survey essentially contains data on the presence of koalas. For the 2006 and 2009-2011 surveys, in addition to information on whether respondents had seen koalas in their area, they were also asked whether they had seen any of a number of widely recognized species. Besides the state-wide koala surveys, a Local Government Area (LGA) public-based survey was also conducted in Bega Valley and Coffs Harbour in 1991, recording only the presence of koalas (Lunney et al., 1997, 2000).

\section{Habitat predictors and threats}

The koala is an obligate folivore, and its diet is restricted mainly to the foliage of Eucalyptus species, restricting its habitat to Eucalyptus dominated forest types (Gordon et al., 1988; Martin \& Handasyde, 1999). In addition, the quality of habitat for the koala can depend on water availability and proximity to permanent water bodies (Gordon et al., 1988; Lunney et al., 2009). Elevation can also affect the quality of Eucalyptus leaves essential for the koala's dietary requirements via a range of environmental factors suitable for koala habitat such as soil quality, rainfall and temperature (Lunney et al., 2009). Therefore, we considered the extent of Eucalyptus forest cover, distance to water features and elevation as key biophysical predictors of koala distributions (sensu Adams-Hosking et al., 2012). Eucalyptus cover ranged from 0 to 100 , where 0 represents no forest cover and 100 represents complete forest cover. We also used mean annual maximum summer temperature and mean annual rainfall as predictors. These variables were chosen because they represent the most climatically influential determinants of Eucalyptus forest distributions (Hughes et al., 1996) and also climatic constraints on koala distributions (Adams-Hosking et al., 2012).

Threats to koalas from vehicle collisions have been studied in detail in NSW and elsewhere, and have been shown to have an important impact on koala populations (Dique et al., 2003; Rhodes et al., 2011; Dudaniec et al., 2013). We therefore generated a spatial variable representing the density of roads to reflect this threat. One way in which the presence of human populations affects koala risk of extinction is through domestic dog attacks, and attacks by domestic dogs are particularly common in urban and peri-urban areas (Dique et al., 2003; Thompson, 2006; Rhodes et al., 2011). As a proxy for the threat from domestically owned dogs, we used housing density.

\section{The occupancy models}

Dynamic occupancy model

We formulated the dynamic occupancy model as described below (sensu MacKenzie et al., 2003). Let $\Psi_{j, 1987}$ denote the probability that grid cell $j$ is occupied by koalas in $1987 ; P_{j, t}$ denote the probability of detecting koalas, given their presence in year $t ; \Omega_{j, t}$ denote the probability that grid cell $j$ is occupied by koalas in survey year $t$ and is unoccupied in year $t+1$ (extinction); and $\Theta_{j, t}$ denote the probability that grid cell $j$ is unoccupied by koalas in survey year $t$ and is occupied in year $t+1$ (colonization). We assumed four census points between 1987 and 2012, each separated by 5 years, that is, $t \in\{1987,1992,2007,2012\}$. Data corresponding to these census points were obtained from the public-based koala surveys conducted in 1987, 1991, 2006 and 2009-2011, respectively, as described in the previous section.

Following Royle \& Kéry (2007), the dynamic occupancy model was formulated within a Bayesian state-space framework, where each observation from respondent $k$ in the $10 \times 10 \mathrm{~km}^{2}$ grid cell $j$ at time $t, y_{k}(j, t)$, was expressed by a two component process: the observations conditional on the unobserved state process, that is, $y_{k}(j, t) \mid z(j, t)$, and the partially observed state process $z(j, t)$. The initial occupancy 
probability $\Psi_{j, 1987}$ was assumed to have a Bernoulli distribution

$$
z(j, 1) \sim \operatorname{Bernoulli}\left(\Psi_{j, 1987}\right) \text { for } j=1, \ldots, n,
$$

whereas the occupancy states in the subsequent periods were assumed to be distributed
We assumed that the colonization rate and probability of detection were constant across space and time, that is, $\Theta_{j, t}=\Theta$ and $P_{t}=P$. We made this assumption because the primary changes through time and the influence of habitat and climatic variables are likely to be related to extinction probability, rather than colonization probability or detectability. In addition, it would be impossible to model explicitly

$$
z(j, t) \mid z(j, t-1) \sim \operatorname{Bernoulli}\left(z(j, t-1)\left(1-\Omega_{j, t-1}\right)+[1-z(j, t-1)] \Theta_{j, t-1}\right)
$$

for $j=1, \ldots, n$ with $n$ denoting the total number of grid cells, and $t=1992,1997, \ldots, 2012$. The observation model for each observation $y_{k}(j, t)$ was then specified conditional on the latent process $z(j, t)$ and given by

$$
y_{k}(j, t) \mid z(j, t) \sim \operatorname{Bernoulli}\left(z(j, t) P_{t}\right) .
$$

In this model, the probability of detecting koalas, $P_{j, t}$, is only identifiable if there are repeat observation events, $y(j, t)$ $z(j, t)$, within each grid cell and within each survey period (MacKenzie et al., 2002, 2003). For survey years containing information on the presence of koalas and the presence of common species (i.e. the 2006 and 2009-2011 public-based surveys), we assumed that the number of observation events within each grid cell was equal to the number of respondents reporting the presence of at least one species in that grid cell, regardless of whether it was a koala or not (sensu Lunney et al., 2009, 2010). The rationale for this is that if respondents had observed at least one common species (even if they had not observed koalas), they were actively observing wildlife and so we considered this to be an observation event. For survey years containing information on the presence of koalas only (i.e. the 1987 and 1991 surveys), we assumed that the number of observation events within each grid cell was the number of respondents reporting the presence of koalas in that grid cell. In grid cells with no observation events, or those grid cells with observation events, but no koalas detected, the true occupancy status of that grid cell, $z(j, t)$, was assumed unobserved. In all other grid cells with observation events, koalas were assumed to be observed, with $z(j, t)=1$.

The probability of a $10 \times 10 \mathrm{~km}^{2}$ grid cell being occupied at the initial survey year, that is, $\Psi_{j, 1987}$, was modelled as a logistic function using the following predictors: elevation, distance to water features, Eucalyptus cover in 1987, housing density in 1987, mean annual maximum summer temperature pre-1987, mean annual rainfall pre-1987, road density and second-order interactions among these variables. The probability of a $10 \times 10 \mathrm{~km}^{2}$ grid cell being occupied at survey year $t$ and unoccupied at survey year $t+1$ (probability of extinction), $\Omega_{j, t}$, was also modelled as a logistic function using the following predictors: Eucalyptus cover, housing density, mean annual maximum summer temperature, mean annual rainfall, road density, all at survey year $t$ and secondorder interactions among these variables. the full spatially colonization process at the scales used in this study. Further, as we had no prior information about detection for each individual survey year, detection probabilities would likely not be identifiable without assuming that they are constant across time. The primary parameters of interest that were estimated for the dynamic occupancy model were the initial occupancy probabilities, $\Psi_{j, 1987}$, the extinction probabilities, $\Omega_{j, t}$, where $t=1992,1997, \ldots, 2012$, the colonization probability $\Theta$, and the detection probability $P$.

\section{Static occupancy model}

Besides the dynamic model described above, we also constructed a competing static occupancy model using only the current 2009-2011 survey data. The primary parameters of interest that were estimated for this static model were the occupancy probabilities, $\Psi_{j, 2011}$ and the detection probability, $P_{2011}$. The probability of occupancy $\Psi_{j, 2011}$ was modelled as a logistic function using the following predictors: elevation, distance to water features, Eucalyptus cover in 2011, housing density in 2011, mean annual maximum summer temperature pre-2011, mean annual rainfall pre2011, road density and second-order interactions among these variables.

\section{Model fitting}

We used WinBUGS Version 1.4.3 (Lunn et al., 2000) to estimate the parameter posterior distributions and the regression coefficients for $\Psi_{j, 1987}, P, \Theta$, and $\Omega_{j, t}$, for $t \in\{1987,1992, \ldots, 2007\}$ in the dynamic occupancy model and for $\Psi_{j, 2011}$ and $P_{2011}$ in the competing static occupancy model. For both the static and dynamic occupancy models, we assumed a uniform prior for each variable explaining occupancy and extinction rates, and for the intercept terms in the colonization and detection probability models. We applied these as informative prior distributions based on the generally known direction of the effect of each predictor variable on koala probability of occurrence and extinction.

We applied a model selection approach to the interaction terms by sequentially adding second-order interaction terms into the initial occupancy component (in 1987) and into the extinction component in the dynamic occupancy model, and then selecting the model with the lowest deviance information criterion (DIC, Spiegelhalter et al., 2002). We applied a 
similar approach to the static occupancy model. This is similar to a standard forward stepwise approach.

For the most parsimonious dynamic occupancy models, we assessed model adequacy using posterior predictive checks (Landwehr et al., 1984; Gelman et al., 2004). We first performed global goodness-of-fit tests, using the deviance as a measure of model fit. We then constructed empirical quantile-quantile plots of the residuals as a graphical assessment of model adequacy. Details of the procedures used for fitting the occupancy models are found in Appendix S2.

As well as estimating the change in occupancy for each $10 \times 10 \mathrm{~km}^{2}$ grid cell within NSW, we also averaged the estimated probabilities of occupancy for each time period for each local government area (LGA), which is the level at which most decisions about koala management are made.

\section{Future scenarios}

We applied our dynamic occupancy model to understand how the distribution of koalas may shift in the future under climate, urban and forest cover change. The aim here was not to make definitive predictions of the future distribution of koalas, but to explore how distributions may change 20 years into the future under a plausible scenario of climate change and the continuation of current trends in habitat loss and household growth. These scenarios are described below and in detail in Appendix S3.

Projected mean annual rainfall and mean annual maximum summer temperature surface maps were obtained from OzClim and provided by the Commonwealth Scientific \& Industrial Research Organisation (CSIRO) (2011). These data were generated based on the CSIRO MK 3.5 climate model and the A1FI high-emission scenario (Gordon et al., 2010). Projected future housing density was modelled based on the historical census data and allowing for contagion in the growth of housing. In doing so, we assumed that historical rates of change in housing density continue into the future. We also developed a scenario of future reduction in vegetation cover caused by human activities, for example, for agriculture, infrastructure and residential developments. This was achieved by assuming that the extent of land clearing in each grid cell follows historical rates of clearing, but modified by increases in the number of households.

\section{RESULTS}

\section{Drivers of koala distributions and extinction risk}

The most parsimonious dynamic occupancy models contained the interaction between the extent of Eucalyptus cover and housing density (Table 1). The second best model was the main effects model (with no interaction term), while the third and fourth best models were those containing the interactions between mean annual maximum summer temperature and Eucalyptus cover or housing density. The differences in DIC between the first ranking model and the
Table 1 Rankings of dynamic occupancy models, pairwise interaction term included in the models, and DIC values for all models within 7 DIC units of the best model. Model rank 2 contains only the main threat variable effects

\begin{tabular}{llll}
\hline Model & $\begin{array}{l}\text { Pairwise interaction } \\
\text { included in the dynamic } \\
\text { rank }\end{array}$ & DIC & $\Delta$ DIC \\
\hline 1 & Eucalyptus cover $\times$ Housing density & 22459.39 & 0.00 \\
2 & - & 22461.43 & 2.04 \\
3 & Eucalyptus cover $\times$ Temperature & 22461.84 & 2.45 \\
4 & Housing density $\times$ Temperature & 22462.31 & 2.92 \\
5 & Temperature $\times$ Rainfall & 22465.95 & 6.56 \\
\hline
\end{tabular}

next three models of the lower rankings were around 2-3 DIC units, indicating some support for the importance of interactions among anthropogenic land use factors on koala distributional change, but most importantly, the interaction between Euclayptus cover and housing density. The goodness-of-fit tests for the most parsimonious dynamic occupancy model showed no significant lack of fit $(P=0.16)$ at a 0.05 significance level. Assessment of the quantile-quantile plots showed some deviation from the expected 1:1 line but, in general, most points lay within the simulated $95 \%$ bounds (Fig. S1). This indicated that the model fitted reasonably well.

For the most parsimonious dynamic occupancy model, the coefficient sizes of the predictors for initial occupancy (in 1987) provide an indication on the magnitude of the effects of climate and environmental predictors on koala long-term occupancy rates (Table 2). The model indicates that mean annual rainfall, distance to water features, and Eucalyptus cover had the largest impact on probability of occupancy. The coefficient sizes of the predictors for the most parsimonious static occupancy model based on 2009-2011 survey data also indicate that mean annual rainfall and distance to water features had the largest impact on probability of occupancy, in agreement with the initial occupancy model. However, the effects of the predictors on extinction risk for the most parsimonious dynamic occupancy model were quite different. The coefficient sizes of the predictors for extinction risk indicate that mean annual maximum summer temperature, Eucalyptus cover, or combined Eucalyptus cover and housing density had the largest impact on extinction risk. Amount of rainfall only had a minimal impact on extinction risk.

Based on the most parsimonious dynamic occupancy model, the probability of occupancy in a $10 \times 10 \mathrm{~km}^{2}$ grid cell was generally higher in the eastern part of NSW than in the western part (Fig. 2a). This is due to higher rainfall and higher Eucalyptus forest cover in the eastern part of NSW. The estimated koala probability of occupancy suggests a rapid decline over the past 20 years. In the period 19871992, the probability of occupancy in each $10 \times 10 \mathrm{~km}^{2}$ grid cell in eastern and western LGAs was approximately 0.7 and 
0.5 , respectively, on average. However, the probability occupancy in a $10 \times 10 \mathrm{~km}^{2}$ grid cell in eastern and western LGAs in 2012 was estimated to be approximately 0.3 and 0.15 , respectively, on average.

Based on the intercept of the extinction model, 5-year koala extinction rates in a $10 \times 10 \mathrm{~km}^{2}$ grid cell across NSW were estimated to be high on average (mean of 0.426 and 95\% credible interval of 0.419-0.433). Similarly, 5-year colonization rates in a $10 \times 10 \mathrm{~km}^{2}$ grid cell were estimated to be low on average (mean of 0.119 and $95 \%$ credible interval of $0.118-0.121)$. Koala populations in western LGAs appear to be generally more susceptible to extinction compared with the populations in eastern LGAs (Fig. 2b). This reflects the fact that the western part of NSW has lower Eucalyptus cover and a higher mean annual maximum summer temperature than the eastern region. In the eastern region, LGAs with high housing density, for example, Sydney and surrounding LGAs, also had high estimated extinction rates.

\section{Future koala distributions and extinction risk under projected environmental change}

Under projected environmental and anthropogenic change, it is estimated that koala probabilities of occupancy in each $10 \times 10 \mathrm{~km}^{2}$ grid cell in the eastern LGAs in 2022 and 2032 will fall to 0.25 and 0.22 , respectively, on average, under our future scenario (Fig. 3a). For western LGAs, the koala probability of occupancy in each $10 \times 10 \mathrm{~km}^{2}$ grid cell in 2022 will fall below 0.1 on average.

For eastern LGAs, with the exception of Sydney and surrounding LGAs, the risk of koalas becoming extinct in a $10 \times 10 \mathrm{~km}^{2}$ grid cell in 2022 and 2032 was estimated to be 0.35 and 0.4 , respectively, on average (Fig. $3 \mathrm{~b}$ ). The risk of koalas becoming extinct in a $10 \times 10 \mathrm{~km}^{2}$ grid cell in the western LGAs by 2022 and 2032 was estimated to be 0.55 and $>0.6$, respectively, on average. The pattern of increase in risk of extinction generally moved gradually over time from the western to the eastern region. This is partly driven by increases in mean annual maximum summer temperatures shifting from the west to the east (Fig. S3). The fact that the western region has lower Eucalyptus forest cover than the eastern region also contributes to this pattern.

\section{DISCUSSION}

The dearth of spatio-temporal species monitoring data over broad spatial extents and over long time periods is a major obstacle for assessing the distributional change of species (Manley et al., 2004; Field et al., 2007). As an alternative, public-based surveys of species distributions can provide a practical and achievable means of collecting this type of data. However, the use of this data to develop dynamic occupancy models over broad extents has received limited attention. Here, we have demonstrated the power of linking publicbased surveys with dynamic occupancy models using data on 
koala populations collected over the past three decades. In particular, this allowed us to understand climatic and habitat drivers of extinction that would not have been possible with the limited field survey data otherwise available, or by taking a static modelling approach. As such, our approach provides a novel and important framework for modelling dynamic change in species' distributions across broad spatial extents.

\section{The modelling approach}

Dynamic occupancy models provide a powerful means of assessing change in species distributions over time and to infer how environmental factors drive these changes based on processes of colonization and extinction. However, to build such models traditionally requires comprehensive field monitoring data of the species over time (Pellet \& Schmidt, 2005). This is a major obstacle for rapidly declining species, where conservation action may be required urgently. For widely distributed species, such as the koala, this poses further chal- lenges, as data on the species must be collected over broad spatial extents. Based on the approach we have developed here, public-based surveys of species can provide a practical means of collecting species occurrence data under time and resource constraints for use in dynamic occupancy models. One of the greatest challenges for doing so is developing detection/non-detection histories from public responses to surveys to enable detection errors to be estimated. We used information on records of other common species to identify non-detections, and this allowed us to construct the detection/non-detection histories (sensu Lunney et al., 2009). Kéry et al. (2010) used a similar approach to this based on data from the Swiss Avian Information Service, but these data were quite different, being field surveys conducted by the public, rather than surveys of the public to obtain sighting information, which are potentially more challenging to deal with. An alternative approach is to construct detection/nondetection histories based on formal interviews to extract information on species sightings (Pillay et al., 2011). (a) 1987-1992

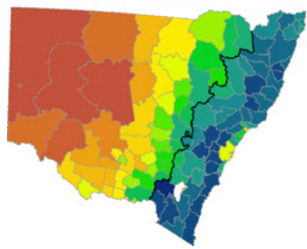

(b) 1987-1992

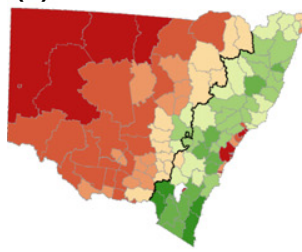

1992-1997

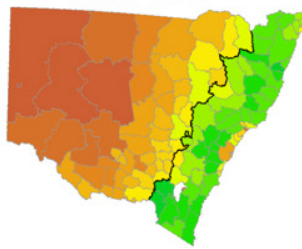

$1992-1997$

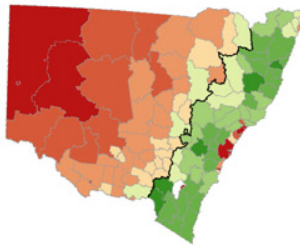

1997-2002

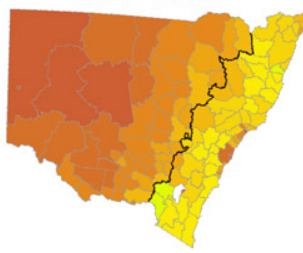

$1997-2002$

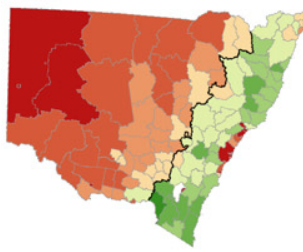

2002-2007

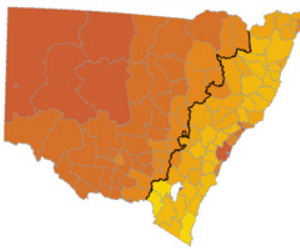

2002-2007

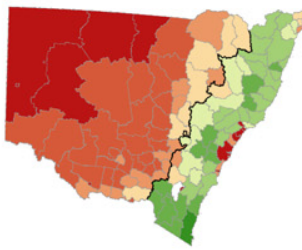

2007-2012

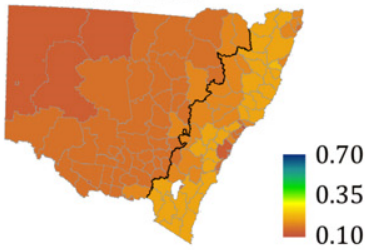

2007-2012

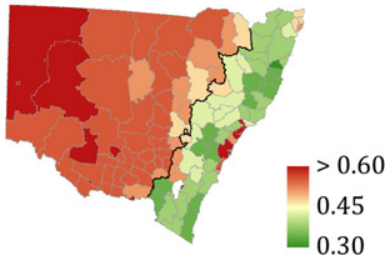

Figure 2 (a) Estimated historical mean koala probabilities of occurrence, and (b) mean extinction rates, for each LGA in NSW. Black line represents the boundary between the western and the eastern LGAs.

(a) 2007-2012

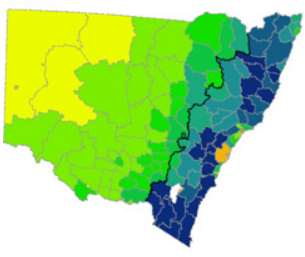

$2017-2022$

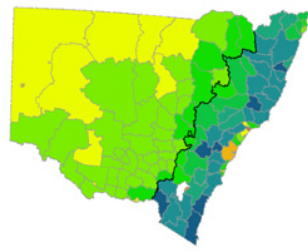

(b)

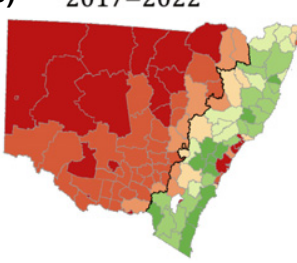

$2027-2032$

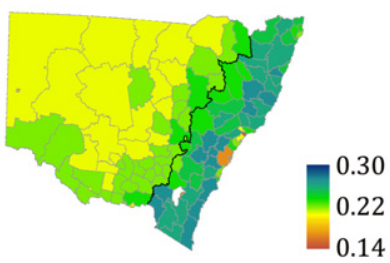

2027-2032

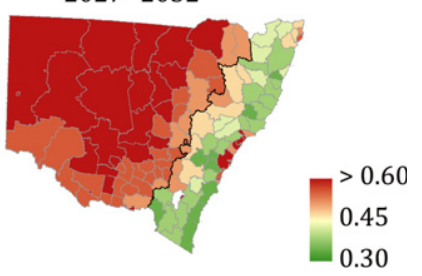

Figure 3 (a) Estimated future koala probabilities of occurrence and (b) risks of extinction, for each LGA in NSW. The map on the far left in (a) was obtained by redrawing the map on the far right in Fig. 2a with different colour levels. Black line represents the boundary between the western and the eastern LGAs. 
However, this approach is likely to be much more resourceintensive than the mail-based survey that the data we used were based on and are likely to be impractical over, very large areas, particularly for species occurring on private lands.

By being able to integrate broad-scale public-based survey data into dynamic occupancy models allowed us to move beyond commonly used methods for predicting species distributions under future climate and environmental change. These are often static and typically use presence-only data collected from community sightings at a single point in time (Elith \& Leathwick, 2009). In particular, we show that, had we only used snapshot data from 2009 to 2011 to understand the drivers of changes in occupancy, we may have introduced considerable bias into our estimates of the effect of environmental change. Based on the static occupancy model derived from the 2009-2011 data, we found that mean annual rainfall and distance to water features had the largest influence on koala probability of occupancy in NSW. However, the drivers of extinction risk in the dynamic occupancy model, the metric that we are predominantly concerned about in conservation, were quite different, and we found that Eucalyptus forest cover and its interaction with housing density had the greatest influence. In this case, annual rainfall only minimally affects the extinction risk of koalas. If inference regarding the relative impact of climate and habitat variables was based on the static occupancy model alone, then this could have misleading implications for recovery planning. Although the shortcomings of the static distribution modelling approaches have previously been pointed out (Zurell et al., 2009), this is one of the first studies to quantify this limitation over broad spatial extents.

In recent years, there has been a recognition that we need to account for dynamic processes in predicting species distributions under future climate and environmental change (Keith et al., 2008; Anderson et al., 2009). So-called coupled models that link static species distribution models with population models have become the favoured approach for addressing this issue. However, they can be data intensive to develop and have inherent uncertainties, particularly with respect to the choice of species distribution model (Pearson et al., 2006; Buisson et al., 2010) and the parameterization of the population model (Dunham et al., 2006). Although dynamic occupancy models are not immune to uncertainties inherited from model input, such as low sample sizes and low species detection (Rota et al., 2009), our approach presents an alternative way for assessing the impacts of climate and land use change on species distributions. A key advantage of our approach of combining dynamic occupancy models with public-based surveys is that the models can be developed cost effectively and, because they are a statistical model, we can importantly obtain statistical measures of uncertainty. Furthermore, the approach could potentially be extended to model community level dynamics using multispecies dynamic occupancy models (Kéry et al., 2009) if public-based surveys could be developed to collect information on multiple species. This would offer a distinct advantage over current approaches.

\section{Limitations and future research}

In the absence of any other information, we assumed constant detection probabilities across grid cells and surveys and this may have introduced some bias into our estimates of occupancy and extinction risk (Rota et al., 2009). Given the decline of koala populations and the species' symbolic status, the probability of detection may have increased over time (e.g. because of its increasing profile). If this is the case, then we may have underestimated detection probability and overestimated occupancy rates and underestimated extinction risk in later years. However, even in the absence of information to estimate variation in detection among surveys (e.g. Kéry et al., 2010), accounting for detection errors is still likely to result in lower bias than if detection errors had been ignored (Kéry, 2011).

A further issue is that the state-wide surveys conducted in 2006 and 2009-2011 applied the same methods and both contained information about the presence of koalas and other common species, but the 1987 state surveys and the 1991 local surveys contain information only about the presence of koalas. To deal with this, we assumed that detection probabilities were the same for these surveys as in later surveys, but that the number of trials in each grid was the same as the number of presences. This would tend to cause an overestimation of detection probability. However, the probability of detection calculated from data from all survey years (mean of 0.51) was found to be very similar to the probability of detection for the 2009-2001 survey data alone (mean of 0.53 ), so it appears that this has little impact on our parameter estimates. Nonetheless, further research to develop ways of incorporating variation in public survey sampling methods through time is important.

We did not explicitly consider koala-habitat responses to climate change in our model, but it is likely that their preferred food tree species will contract towards the east coast as a result of climate change over the next 50 years (AdamsHosking et al., 2012). This means that the impact of climate change may have been underestimated to some extent. Explicitly incorporating future shifts in the distributions of Eucalyptus may improve model predictions, particularly in the western LGAs where climate conditions may become unsuitable for many koala habitat tree species (AdamsHosking et al., 2012). Furthermore, we did not consider the fact that there may be spatial variation in the effect of landscape and climate change on koalas across the study region. It has been shown that koala-habitat relationships can vary substantially from region to region (McAlpine et al., 2008; Rhodes et al., 2008), so this may be an important factor that drives spatial variation in responses across NSW. Therefore, our model could be improved by explicitly incorporating spatial variation (non-stationarity) in these relationships across spatial scales (Hochachka et al., 2012). However, additional data collected at a range of scales may be necessary to enable this. 


\section{CONCLUSIONS}

This study demonstrates the value of combining public surveys and dynamic occupancy modelling to assess species risk of extinction and help to identify priority areas requiring conservation action due to pressing climate and anthropogenic threats. Our approach is a significant advance over the conventional static species distribution modelling approaches. Our approach also presents an alternative to the coupled species distribution model and population model approach by allowing explicit estimation of the importance of environmental predictors on extinction probability and a formal estimation of statistical measures of uncertainty. Although static species distribution models will continue to be used to inform conservation decisions under future climate change scenarios when long-term monitoring data on species' occurrence are not readily available, well-designed public-based surveys in the future should allow for increasing development of dynamic models.

\section{ACKNOWLEDGEMENTS}

We thank Elly Stalenberg, Rod Ruffio, Karen Borchardt, Ian Shannon, Mathew Crowther and Steven Cordwell for assistance with the data, and three anonymous referees for constructive comments. This research was conducted with the support of funding from the Australian Government's National Environmental Research Program.

\section{REFERENCES}

Adams-Hosking, C., McAlpine, C., Rhodes, J.R., Grantham, H.S. \& Moss, P.T. (2012) Modelling changes in the distribution of the critical food resources of a specialist folivore in response to climate change. Diversity and Distributions, 18, 847-860.

Anderson, B., Akçakaya, H., Araújo, M., Fordham, D., Martinez-Meyer, E., Thuiller, W. \& Brook, B. (2009) Dynamics of range margins for metapopulations under climate change. Proceedings of the Royal Society B: Biological Sciences, 276, 1415-1420.

Bellard, C., Bertelsmeier, C., Leadley, P., Thuiller, W. \& Courchamp, F. (2012) Impacts of climate change on the future of biodiversity. Ecology Letters, 15, 365-377.

Buisson, L., Thuiller, W., Casajus, N., Lek, S. \& Grenouillet, G. (2010) Uncertainty in ensemble forecasting of species distribution. Global Change Biology, 16, 1145-1157.

Commonwealth Scientific and Industrial Research Organisation (CSIRO) (2011) OzClim: A Climate Scenario Generator and Impacts Package for Australia. (web download), Available at: http://www.csiro.au/ozclim/home.do (accessed 24 July 2011).

Conlisk, E., Syphard, A.D., Franklin, J., Flint, L., Flint, A. \& Regan, H. (2013) Uncertainty in assessing the impacts of global change with coupled dynamic species distribution and population models. Global Change Biology, 19, 858-869.
Devictor, V., Whittaker, R.J. \& Beltrame, C. (2010) Beyond scarcity: citizen science programmes as useful tools for conservation biogeography. Diversity and Distributions, 16, 354-362.

Dique, D.S., Thompson, J., Preece, H.J., Penfold, G.C., de Villiers, D.L. \& Leslie, R.S. (2003) Koala mortality on roads in southeast Queensland: the koala speed-zone trial. Wildlife Research, 30, 419-426.

Dudaniec, R.Y., Rhodes, J.R., Worthington Wilmer, J., Lyons, M., Lee, K.E., McAlpine, C.A. \& Carrick, F.N. (2013) Using multilevel models to identify drivers of landscape-genetic structure among management areas. Molecular Ecology, 22, 3752-3765.

Dunham, A.E., Akćakaya, H.R. \& Bridges, T.S. (2006) Using scalar models for precautionary assessments of threatened species. Conservation Biology, 20, 1499-1506.

Elith, J. \& Leathwick, J.R. (2009) Species distribution models: ecological explanation and prediction across space and time. Annual Review of Ecology, Evolution, and Systematics, 40, 677-697.

Field, S.A., O’Connor, P.J., Tyre, A.J. \& Possingham, H.P. (2007) Making monitoring meaningful. Austral Ecology, 32, 485-491.

Gelman, A., Carlin, J.B., Stern, H.S. \& Rubin, D.B. (2004) Bayesian data analysis. Chapman \& Hall/CRC, Boca Raton, FL.

Gordon, G., Brown, A. \& Pulsford, T. (1988) A Koala (Phascolarctos cinereus Goldfuss) population crash during drought and heatwave conditions in south-western Queensland. Australian Journal of Ecology, 13, 451-461.

Gordon, H., O’Farrell, S., Collier, M., Dix, M., Rotstayn, L., Kowalczyk, E., Hirst, T. \& Watterson, I. (2010) The CSIRO Mk3.5 climate model. CAWCR Technical Report 21. The Centre for Australian Weather and Climate Research, Aspendale, Australia.

Hochachka, W.M., Fink, D., Hutchinson, R.A., Sheldon, D., Wong, W.K. \& Kelling, S. (2012) Data-intensive science applied to broad-scale citizen science. Trends in Ecology \& Evolution, 27, 130-137.

Hughes, L., Cawsey, E.M. \& Westoby, M. (1996) Climatic range sizes of Eucalyptus species in relation to future climate change. Global Ecology and Biogeography Letters, 5, 23-29.

Keith, D.A., Akçakaya, H.R., Thuiller, W., Midgley, G.F., Pearson, R.G., Phillips, S.J., Regan, H.M., Araújo, M.B. \& Rebelo, T.G. (2008) Predicting extinction risks under climate change: coupling stochastic population models with dynamic bioclimatic habitat models. Biology Letters, 4, 560-563.

Kéry, M. (2011) Towards the modelling of true species distributions. Journal of Biogeography, 38, 617-618.

Kéry, M., Royle, J.A., Plattner, M. \& Dorazio, R.M. (2009) Species richness and occupancy estimation in communities subject to temporary emigration. Ecology, 90, 1279-1290.

Kéry, M., Royle, J.A., Schmid, H., Schaub, M., Volet, B., Haefliger, G. \& Zbinden, N. (2010) Site-occupancy distribution modeling to correct population-trend estimates derived from opportunistic observations. Conservation Biology, 24, 1388-1397. 
Landwehr, J.M., Pregibon, D. \& Shoemaker, A.C. (1984) Graphical methods for assessing logistic regression models. Journal of the American Statistical Association, 79, 61-71.

Lunn, D.J., Thomas, A., Best, N. \& Spiegelhalter, D. (2000) WinBUGS - a Bayesian modelling framework: concepts, structure, and extensibility. Statistics and Computing, 10, 325-337.

Lunney, D., Esson, C., Moon, C., Ellis, M. \& Matthews, A. (1997) A community-based survey of the koala, Phascolarctos cinereus, in the Eden region of south-eastern New South Wales. Wildlife Research, 24, 111-128.

Lunney, D., Matthews, A., Moon, C. \& Ferrier, S. (2000) Incorporating habitat mapping into practical koala conservation on private lands. Conservation Biology, 14, 669-680.

Lunney, D., Crowther, M.S., Shannon, I. \& Bryant, J.V. (2009) Combining a map-based public survey with an estimation of site occupancy to determine the recent and changing distribution of the koala in New South Wales. Wildlife Research, 36, 262-273.

Lunney, D., Close, R., Crowther, M.S., Bryant, J., Shannon, I., Madden, K. \& Ward, S. (2010) The koalas of Campbelltown, south-western Sydney: does their natural history foretell of an unnatural future? The natural history of Sydney (ed. by D. Lunney, P. Hutchings and D. Hochuli), pp. 339-370. Royal Zoological Society of NSW, Mosman, NSW.

Lunney, D., Stalenberg, E., Santika, T. \& Rhodes, J.R. (in press) Extinction in Eden: identifying the role of climate change in the decline of the koala in south-eastern NSW. Wildlife Research.

MacKenzie, D.I., Nichols, J.D., Lachman, G.B., Droege, S., Royle, J.A. \& Langtimm, C.A. (2002) Estimating site occupancy rates when detection probabilities are less than one. Ecology, 83, 2248-2255.

MacKenzie, D.I., Nichols, J.D., Hines, J.E., Knutson, M.G. \& Franklin, A.B. (2003) Estimating site occupancy, colonisation, and local extinction when a species is detected imperfectly. Ecology, 84, 2200-2207.

Mackey, B.G. \& Lindenmayer, D.B. (2001) Towards a hierarchical framework for modelling the spatial distribution of animals. Journal of Biogeography, 28, 1147-1166.

Manley, P.N., Zielinski, W.J., Schlesinger, M.D. \& Mori, S.R. (2004) Evaluation of a multiple-species approach to monitoring species at the ecoregional scale. Ecological Applications, 14, 296-310.

Mantyka-Pringle, C.S., Martin, T.G. \& Rhodes, J.R. (2012) Interactions between climate and habitat loss effects on biodiversity: a systematic review and meta-analysis. Global Change Biology, 18, 1239-1252.

Martin, R. \& Handasyde, K. (1999) The koala: natural history, conservation and management. UNSW Press, Sydney, NSW.

Martin, J., McIntyre, C.L., Hines, J.E., Nichols, J.D., Schmutz, J.A. \& MacCluskie, M.C. (2009) Dynamic multistate site occupancy models to evaluate hypotheses relevant to conservation of Golden Eagles in Denali National Park, Alaska. Biological Conservation, 142, 2726-2731.

McAlpine, C.A., Rhodes, J.R., Bowen, M.E., Lunney, D., Callaghan, J.G., Mitchell, D.L. \& Possingham, H.P. (2008)
Can multi-scale models of a species' distribution be generalised from region to region? A case study of the koala. Journal of Applied Ecology, 45, 558-567.

Moilanen, A., Smith, A.T. \& Hanski, I. (1998) Long-term dynamics in a metapopulation of the American pika. The American Naturalist, 152, 530-542.

Pearson, R.G. \& Dawson, T.P. (2003) Predicting the impacts of climate change on the distribution of species: are bioclimate envelope models useful? Global Ecology and Biogeography, 12, 361-371.

Pearson, R.G., Thuiller, W., Araújo, M.B., Martinez-Meyer, E., Brotons, L., McClean, C., Miles, L., Segurado, P., Dawson, T.P. \& Lees, D.C. (2006) Model-based uncertainty in species range prediction. Journal of Biogeography, 33, 1704-1711.

Pellet, J. \& Schmidt, B.R. (2005) Monitoring distributions using call surveys: estimating site occupancy, detection probabilities and inferring absence. Biological Conservation, 123, 27-35.

Pillay, R., Johnsingh, A.J.T., Raghunatha, R. \& Madhusudan, M.D. (2011) Patterns of spatiotemporal change in large mammal distribution and abundance in the southern Western Ghats, India. Biological Conservation, 144, 15671576.

Reed, P.C., Lunney, D. \& Walker, P. (1990) A 1986-1987 survey of the koala Phascolarctos cinereus (Goldfuss) in New South Wales and an ecological interpretation of its distribution. Biology of the koala (ed. by A.K. Lee, K.A. Handasyde and G.D. Sanson), pp. 55-74. Surrey Beatty and Sons, Sydney.

Rhodes, J.R., Callaghan, J.G., McAlpine, C.A., de Jong, C., Bowen, M.E., Mitchell, D.L., Lunney, D. \& Possingham, H.P. (2008) Regional variation in habitat-occupancy thresholds: a warning for conservation planning. Journal of Applied Ecology, 45, 549-557.

Rhodes, J.R., Ng, C.F., de Villiers, D.L., Preece, H.J., McAlpine, C.A. \& Possingham, H.P. (2011) Using integrated population modelling to quantify the implications of multiple threatening processes for a rapidly declining population. Biological Conservation, 144, 1081-1088.

Rota, C.T., Fletcher, R.J. Jr, Dorazio, R.M. \& Betts, M.G. (2009) Occupancy estimation and the closure assumption. Journal of Applied Ecology, 46, 1173-1181.

Royle, J.A. \& Kéry, M. (2007) A Bayesian state-space formulation of dynamic occupancy models. Ecology, 88, 1813-1823.

Spiegelhalter, D.J., Best, N.G., Carlin, B.P. \& Van Der Linde, A. (2002) Bayesian measures of model complexity and fit. Journal of the Royal Statistical Society: Series B (Statistical Methodology), 64, 583-639.

Thompson, J. (2006) The Comparative Ecology and Population Dynamics of Koalas in the Koala Coast Region of South-East Queensland. PhD Thesis, The University of Queensland, Brisbane, Australia.

Thuiller, W., Albert, C., Araujo, M.B., Berry, P.M., Cabeza, M., Guisan, A., Hickler, T., Midgely, G.F., Paterson, J., Schurr, F.M., Sykes, M.T. \& Zimmermann, N.E. (2008) Predicting global change impacts on plant species' 
distributions: future challenges. Perspectives in Plant Ecology Evolution and Systematics, 9, 137-152.

Van der Hoeven, C.A., De Boer, W.F. \& Prins, H.H.T. (2004) Pooling local expert opinions for estimating mammal densities in tropical rainforests. Journal for Nature Conservation, 12, 193-204.

Wilson, K.A., Pressey, R.L., Newton, A., Burgman, M., Possingham, H. \& Weston, C. (2005) Measuring and incorporating vulnerability into conservation planning. Environmental Management, 35, 527-543.

Zurell, D., Jeltsch, F., Dormann, C.F. \& Schroder, B. (2009) Static species distribution models in dynamically changing systems: how good can predictions really be? Ecography, 32, 733-744.

\section{SUPPORTING INFORMATION}

Additional Supporting Information may be found in the online version of this article:

Appendix S1 Data on koala, habitat predictors and threats.
Appendix S2 Fitting the occupancy models.

Appendix S3 Defining future scenarios.

Figure S1 Quantile-quantile plots for the most parsimonious dynamic occupancy model.

\section{BIOSKETCH}

Truly Santika is interested in the modelling of species distributions, population dynamics and spatio-temporal prioritization for biodiversity conservation and natural resource management.

Author contributions: T.S, C.A.M, D.L, K.A.W and J.R.R conceived the research ideas, D.L contributed the koala data; T.S analysed the data and carried out the modelling, T.S led the writing, C.A.M, D.L, K.A.W and J.R.R contributed to data analysis, modelling and writing.

Editor: Wilfried Thuiller 